\title{
Coordinated Orbit Transfer for Satellite Clusters
}

\author{
FUMIN ZHANG AND P.S. KRISHNAPRASAD \\ Institute for Systems Research and Department of Electrical and Computer Engineering, \\ University of Maryland at College Park, College Park, Maryland, USA
}

\begin{abstract}
We propose a control law that allows a satellite formation to achieve orbit transfer. During the transfer, the formation can be either maintained or modified to a desired formation. Based on the orbit transfer control law proposed by Chang, Chichka, and Marsden for single satellite, we add coupling terms to the summation of Lyapunov functions for single satellites. These terms are functions of the difference between the mean anomalies (or perigee passage times) of formation members. The asymptotic stability of the desired formation in desired orbits is proved.
\end{abstract}

KEYWORDS: momentum map; control Lyapunov function; formation control; orbit transfer

\section{INTRODUCTION}

This paper is concerned with the problem of achieving a satellite formation near a designated elliptic orbit. For orbits near the earth, one can use a space shuttle to place satellites into specified relative positions. What we want to consider here is the case when members of a cluster have been placed relatively far apart. They have to use their on-board thrusters to get to the desired orbits and achieve the desired formation. A similar case is when the entire formation needs to be restructured for mission-related reasons. We want the formation to be maintained to some extent during the transfer and be reestablished after the transfer.

Our approach is to use Lyapunov functions to design the control laws for orbit transfer. The Lyapunov function achieves a local minimum when the correct orbit and formation are reached. In Reference 1, a Lyapunov function is expressed as a quadratic function of the differences of orbital elements between current orbit and the destination orbit. However, in that work convergence of the associated control law is not proved.

In this paper, we develop new control laws for orbit transfer of formations and give a proof of convergence. This algorithm is based on a Lyapunov function on the shape space of elliptic orbits in our previous work, ${ }^{2}$ the work of Chang, Chichka, and Marsden, ${ }^{3}$ and a result of Cushman and Bates. ${ }^{4}$ However, the most significant extension is the addition of a coupling term that is a function of the difference between

Address for correspondence: P.S. Krishnaprasad, Institute for Systems Research and Department of Electrical and Computer Engineering, University of Maryland at College Park, College Park, MA 20742, USA.

krishna@isr.umd.edu

Ann. N.Y. Acad. Sci. 1017: 112-137 (2004). (C2004 New York Academy of Sciences. doi: 10.1196/annals.1311.008 
the osculating perigee passage times. For circular orbits, we use the ascending node passage time instead of the perigee passage time.

In the next section, we develop formulæ used in the proofs of our theorems. Thereafter, we give a brief summary of results published elsewhere. ${ }^{2,3}$ are given. We then introduce the definition of periodic satellite formations, followed by our main results and proofs about orbit transfer of periodic formations. Simulation results are provided in the final section of our paper.

\section{CONTROL OSCULATING ELEMENTS}

If the mass of a satellite is small compared to the mass of the Earth, the Kepler two body problem can be approximated by a one center problem as follows:

$$
m \ddot{q}=-\nabla V_{G}+u,
$$

where $q \in \mathbb{R}^{3}$ is the position vector of the satellite relative to the center of the Earth, $m$ is the mass of the satellite, $V_{G}$ is the gravitational potential of the Earth, $u$ is the control force plus other disturbances. Without considering higher order terms, $V_{G}$ takes the form

$$
V_{G}=-m \frac{\mu}{\|q\|}
$$

Let $p=m \dot{q}$ be the momentum vector of the satellite. For simplicity we assume that all the satellites considered in this paper have unit mass.

We define the following:

$$
\begin{aligned}
l(t) & =q(t) \times p(t) \\
A(t) & =p(t) \times l(t)-\mu \frac{q(t)}{\|q(t)\|} \\
e(t) & =\frac{\|A(t)\|}{\mu} \\
a(t) & =\frac{h(t)^{2}}{\mu\left(1-e(t)^{2}\right)} \\
\cos (E(t)) & =\frac{1}{e(t)}\left(1-\frac{r(t)}{a(t)}\right) \\
M(t) & =E(t)-e(t) \sin (E(t)),
\end{aligned}
$$

where $h(t)=\|l(t)\|$ and $r(t)=\|q(t)\|$ (these formulæ can be found in textbooks on celestial mechanics $\left.{ }^{5}\right) ; l$ is the angular momentum vector and $A$ is called the Laplace vector (both are conserved if $u(t) \equiv 0$ ); $a$ is the length of the semimajor axis; $e$ is the eccentricity; $E$ is the eccentric anomaly; and $M$ is the mean anomaly. The last equation is Kepler's equation. When $e(t)=0$, the eccentric anomaly $E(t)$ is defined to be $M(t)$, which is measured from the line of ascending node. For now, we assume that $e(t) \neq 0$.

Note that these formulæ are valid for all $t$ and all the elements are differentiable on $\mathbb{R}^{3} \times \mathbb{R}^{3}-\{0\}$. Hence, we can take derivative on both sides of Equations (3). By using the property that $l, A, a$, and $e$ are conserved when $u(t) \equiv 0$, we have 


$$
\begin{aligned}
\dot{l}(t) & =\frac{\partial l}{\partial q} \dot{q}+\frac{\partial l}{\partial p} \dot{p} \\
& =\frac{\partial l}{\partial q} \dot{q}+\frac{\partial l}{\partial p}\left(-\nabla V_{G}+u\right) \\
& =\left.i\right|_{u=0}+\frac{\partial l}{\partial p} u(t) \\
& =\frac{\partial l}{\partial p} u(t) .
\end{aligned}
$$

Note that we used the property that $l$ is conserved when $u(t) \equiv 0$. Similarly,

$$
\dot{A}(t)=\frac{\partial A}{\partial p} u(t) \text {. }
$$

We obtain the derivatives of the orbital elements in the APPENDIX, the results are listed below

$$
\dot{e}(t)=\frac{1}{\mu}\left(\frac{\partial A}{\partial p}\right)^{T} \hat{A} \cdot u(t),
$$

where $\hat{A}$ is the unit vector along the direction of $A$. We also have

$$
\begin{gathered}
\dot{a}(t)=\frac{2}{\mu\left(1-e^{2}\right)}\left(\frac{\partial l}{\partial p}\right)^{T} l \cdot u(t)+\frac{2 a e}{\mu\left(1-e^{2}\right)}\left(\frac{\partial A}{\partial p}\right)^{T} \hat{A} \cdot u(t) \\
\dot{E}=\sqrt{\frac{\mu}{a}} \frac{1}{r}+\frac{\cos (E)}{e \sin (E)} \dot{e}-\frac{r}{a^{2} e \sin (E)} \dot{a} \\
\dot{M}=n+\eta\left(\frac{\partial A}{\partial p}\right)^{T} \hat{A} \cdot u(t)+\xi\left(\frac{\partial l}{\partial p}\right)^{T} l \cdot u(t),
\end{gathered}
$$

where $n=\sqrt{\mu / a^{3}}$ and

$$
\begin{aligned}
\xi(l, A, E) & =-\frac{2(1-e \cos (E))^{2}}{\mu a e\left(1-e^{2}\right) \sin (E)} \\
\eta(l, A, E) & =\frac{\cos ((E)-e)}{\mu e \sin (E)}-\frac{2(1-e \cos (E))^{2}}{\mu\left(1-e^{2}\right) \sin E} .
\end{aligned}
$$

Note that $\xi$ and $\eta$ are infinite if $\sin (E)=0$. Physically, this means that when the satellite is passing perigee or apogee, the control can cause a sudden jump of the mean and eccentric anomalies. To prevent this from happening in our control laws, we turn off the control when $\sin (E)=0$.

\section{ORBIT TRANSFER OF SINGLE SATELLITE}

For a single satellite on an elliptic orbit, the set $D$ of ordered pairs $(l, A)$ is a subset of $\mathbb{R}^{3} \times \mathbb{R}^{3}$ with Euclidean norm,

$$
D=\left\{(l, A) \in \mathbb{R}^{3} \times \mathbb{R}^{3} \mid A \cdot l=0, l \neq 0,\|A\|<m^{2} \mu\right\} .
$$

Let $W=\frac{1}{2} m\|\dot{q}\|^{2}+V_{G}$ be the total energy of the satellite. Let $P$ be the set of all pairs $(q, p)$ with Euclidean norm. Then, we define a set $\Sigma_{e}$ by 


$$
\Sigma_{e}=\{(q, p) \in P \mid W(q, p)<0, l \neq 0\} .
$$

By definition of $l$ and $A$, we have a mapping $\pi: \Sigma_{e} \rightarrow D,(q, p) \mapsto(l, A)$.

Theorem 1: (Chang-Chichka-Marsden $)^{3}$

The following hold:

1. $\Sigma_{e}$ is the union of all elliptic Keplerian orbits.

2. $\pi\left(\Sigma_{e}\right)=D$ and $\Sigma_{e}=\pi^{-1}(D)$.

3. The fiber $\pi^{-1}(l, A)$ is a unique (oriented) elliptic Keplerian orbit for each

$(l, A) \in D$ (see also Ref. 4, page 58).

The mapping $\pi$ is a continuous mapping because $(l, A)$ are continuous with respect to $(q, p)$.

Corollary 2:

$\pi^{-1}(K)$ is compact for any compact set $K \subset D$ (c.f., Ref. 2).

If we have $n \geq 1$ satellites, let $\bar{D}=D_{1} \times D_{2} \times \ldots \times D_{n}$ and let

$$
\bar{d}=d_{1 \mid D_{1}}+d_{\left.2\right|_{D_{2}}}+\ldots+d_{\left.n\right|_{n}} .
$$

We define the shape space of elliptic Keplerian orbits to be the set $\bar{D}$ with the distance function $\bar{d}$. The above theorem and corollary can be extended to $(\bar{D}, \bar{d})$.

To control the orbit transfer of a single satellite, one considers a Lyapunov function ${ }^{3}$

$$
V(q, p)=\frac{1}{2}\left(\left\|l-l_{d}\right\|^{2}+\left\|A-A_{d}\right\|^{2}\right),
$$

where $\left(l_{d}, A_{d}\right)$ is the pair of the angular momentum vector and Laplace vector of the target elliptic (circular) orbit. The derivative of $V$ along the integral curve of the system is

$$
\begin{aligned}
\dot{V} & =\left(l-l_{d}\right) \cdot \dot{l}+\left(A-A_{d}\right) \cdot \dot{A} \\
& =\left(l-l_{d}\right) \cdot \frac{\partial l}{\partial p} u+\left(A-A_{d}\right) \cdot \frac{\partial A}{\partial p} u \\
& =\left[\left(l-l_{d}\right) \times q+l \times\left(A-A_{d}\right)+\left(A-A_{d}\right) \times p \times q\right] \cdot u .
\end{aligned}
$$

If we let the control be

$$
u=-\left[\left(l-l_{d}\right) \times q+l \times\left(A-A_{d}\right)+\left(\left(A-A_{d}\right) \times p\right) \times q\right],
$$

then $\dot{V} \leq 0$ along the trajectory of the closed loop system. The following theorem is proved.

Theorem 3: (Chang-Chichka-Marsden)

There exists $c>0$ such that if $V\left(q_{0}, p_{0}\right) \leq c$, by applying the control law in Equation (15), the trajectory of the closed loop system starting at $\left(q_{0}, p_{0}\right)$ will asymptotically converge to the target orbit $\pi^{-1}\left(l_{d}, A_{d}\right)$. 


\section{PERIODIC FORMATION}

Suppose we have a formation consisting of $m$ satellites. Let $O_{j}$ denote the orbit of the $j$ th satellite. The orbit $O_{j}$ and the position of the satellite on $O_{j}$ can be described either by the six orbital elements $\left(a_{j}, e_{j}, I_{j}, \Omega_{j}, \omega_{j}, \tau_{j}\right)$ or by $\left(l_{j}, A_{j}, \tau_{j}\right)$ where $a$ denotes the length of semimajor axis, $e$ is the eccentricity, $I$ is the inclination, $\Omega$ is the longitude of the ascending node, $\omega$ is the argument of the perigee, and $\tau$ is the perigee passage time. If $O_{j}$ is elliptic or circular, except for singular cases for which some of the orbital elements are not well defined, Theorem 1 tells us that the two expressions are equivalent. Among all possible formations, we are interested in formations with periodic shape changes. We introduce the following definition.

\section{Definition 1:}

A formation is periodic when $a_{j}$, the length of semimajor axis of orbit $O_{j}$, satisfies $a_{j}=a>0$ for all $j=1,2, \ldots, m$.

This definition is valid since all the satellites in a periodic formation have the same orbital period

$$
T=\frac{2 \pi \sqrt{a^{3}}}{\sqrt{\mu}} .
$$

Thus, although the shape of the formation is varying, it is varying periodically.

On the other hand, given a set of orbits with identical length semimajor axis, there are infinitely many possible periodic formations. We need to specify their relative positions on the orbits to determine a specific formation. The difficulty is that the relative positions are complicated functions of time. However, the differences between the perigee passage times, $\left(\tau_{i}-\tau_{j}\right)$, are constants. Because the mean anomaly is

$$
M_{i}=n_{i}\left(t-\tau_{i}\right),
$$

where $n_{i}=2 \pi / T$, then $\left(M_{i}-M_{j}\right)$ are constants. By specifying the values of $\left(\tau_{i}-\tau_{j}\right)$ or $\left(M_{i}-M_{j}\right)$ for all $i$ and $j$, a periodic formation can be uniquely determined.

\section{TRANSFER OF SATELLITE FORMATIONS BETWEEN ELLIPTIC ORBITS}

With all the tools we need developed, we are now ready to determine our control laws. To illustrate our ideas, we show the calculations for two satellites. A control law for more than two satellites can be developed based on the two-satellite case in a natural way.

To set up a periodic formation of two satellites, one can control each satellite separately to transfer to its target orbit. However, this will not assure the correct values for $\left(\tau_{i}-\tau_{j}\right)$ or $\left(M_{i}-M_{j}\right)$. To do that, additional terms involving $\left(\tau_{i}-\tau_{j}\right)$ or $\left(M_{i}-M_{j}\right)$ should be added in the summation of the Lyapunov functions for single satellites. This extension results in a cooperative orbit transfer of multiple satellites.

We introduce a variable $\Upsilon_{i}$, defined by 


$$
\Upsilon_{i}=\left\{\begin{array}{l}
\left(\frac{a_{i}}{a_{d}}\right)^{3 / 2} M_{i}, \text { if } E_{i} \in(-\varepsilon, \pi+\varepsilon) \\
\left(\frac{a_{i}}{a_{d}}\right)^{3 / 2}\left(2 \pi-M_{i}\right), \text { if } E_{i} \in(\pi-\varepsilon, 2 \pi+\varepsilon)
\end{array}\right.
$$

for $i=1,2$, where $a_{d}$ is the common length of the semimajor axes for the destination orbits.

Here, the trouble of using different expressions for the cases $E_{i} \in(-\varepsilon, \pi+\varepsilon)$ and $E_{i} \in(\pi-\varepsilon, 2 \pi+\varepsilon)$ is caused by the fact that $E_{i} \in S^{1}$ a circle. Two coordinate charts are required on $S^{1}$. Here we pick the charts to be

$$
\begin{aligned}
& \psi_{1}:(-\varepsilon, \pi+\varepsilon) \rightarrow(-\varepsilon, \pi+\varepsilon) \text { s.t. } E_{i} \mapsto E_{i} \\
& \psi_{2}:(\pi-\varepsilon, 2 \pi+\varepsilon) \rightarrow(-\varepsilon, \pi+\varepsilon) \text { s.t. } E_{i} \mapsto\left(2 \pi-E_{i}\right) .
\end{aligned}
$$

The value of $\varepsilon$ is chosen so that the two satellites are in the same chart. Because in a satellite formation the angular separations between satellites are usually small, the value of $\varepsilon$ is small.

For $E_{i} \in(-\varepsilon, \pi+\varepsilon)$, we have

$$
\begin{aligned}
\dot{\Upsilon}_{i} & =\left(\frac{a_{i}}{a_{d}}\right)^{3 / 2} \dot{M}_{i}+\frac{3}{2} \sqrt{\frac{a_{i}}{a_{d}^{3}}} \dot{a}_{i} M_{i} \\
& =\sqrt{\frac{\mu}{a_{d}^{3}}}+\rho\left(l_{i}, A_{i}, E_{i}\right)\left(\frac{\partial A_{i}}{\partial p_{i}}\right)^{T} \hat{A}_{i} \cdot u_{i}(t)+\zeta\left(l_{i}, A_{i}, E_{i}\right)\left(\frac{\partial l_{i}}{\partial p_{i}}\right)^{T} l_{i} \cdot u_{i}(t),
\end{aligned}
$$

where

$$
\begin{aligned}
& \zeta=\left(\frac{a_{i}}{a_{d}}\right)^{3 / 2} \xi\left(l_{i}, A_{i}, E_{i}\right)+\frac{3}{2} \sqrt{\frac{a_{i}}{a_{d}^{3}}} \frac{2}{\mu\left(1-e_{i}^{2}\right)} M_{i} \\
& \rho=\left(\frac{a_{i}}{a_{d}}\right)^{3 / 2} \eta\left(l_{i}, A_{i}, E_{i}\right)+\frac{3}{2} \sqrt{\frac{a_{i}}{a_{d}^{3}}} \frac{2 a_{i} e_{i}}{\mu\left(1-e_{i}^{2}\right)} M_{i} .
\end{aligned}
$$

For $E_{i} \in(\pi-\varepsilon, 2 \pi+\varepsilon)$,

$$
\begin{aligned}
\dot{\Upsilon}_{i} & =-\left(\frac{a_{i}}{a_{d}}\right)^{3 / 2} \dot{M}_{i}+\frac{3}{2} \sqrt{\frac{a_{i}}{a_{d}^{3}}} \dot{a}_{i}\left(2 \pi-M_{i}\right) \\
& =-\sqrt{\frac{\mu}{a_{d}^{3}}}+\rho\left(l_{i}, A_{i}, E_{i}\right)\left(\frac{\partial A_{i}}{\partial p_{i}}\right)^{T} \hat{A}_{i} \cdot u_{i}(t)+\zeta\left(l_{i}, A_{i}, E_{i}\right)\left(\frac{\partial l_{i}}{\partial p_{i}}\right)^{T} l_{i} \cdot u_{i}(t),
\end{aligned}
$$

where

$$
\begin{aligned}
& \zeta=-\left(\frac{a_{i}}{a_{d}}\right)^{3 / 2} \xi\left(l_{i}, A_{i}, E_{i}\right)+\frac{3}{2} \sqrt{\frac{a_{i}}{a_{d}^{3}}} \frac{2}{\mu\left(1-e_{i}^{2}\right)}\left(2 \pi-M_{i}\right) \\
& \rho=-\left(\frac{a_{i}}{a_{d}}\right)^{3 / 2} \eta\left(l_{i}, A_{i}, E_{i}\right)+\frac{3}{2} \sqrt{\frac{a_{i}}{a_{d}^{3}}} \frac{2 a_{i} e_{i}}{\mu\left(1-e_{i}^{2}\right)}\left(2 \pi-M_{i}\right) .
\end{aligned}
$$


Note that we have terms that explicitly contain $M_{i}$. If not handled well, these terms cause discontinuity in our control algorithm when the satellites enter a new chart. The reason for us to pick the particular charts $\left(\psi_{1}, \psi_{2}\right)$ is to reduce the discontinuities in the derivatives of $\Upsilon_{i}$ caused by changing charts.

We design a Lyapunov function on the phase space of the two satellites. This single function has different expressions in different charts. The Lyapunov function is

$$
V=\left\{\begin{array}{l}
V_{1}+V_{2}+4 \sin \left(\frac{\Upsilon_{1}-\Upsilon_{2}-\phi}{4}\right)^{2}, \text { if } E_{i} \in(-\varepsilon, \pi+\varepsilon) \\
V_{1}+V_{2}+4 \sin \left(\frac{\Upsilon_{1}-\Upsilon_{2}+\phi}{4}\right)^{2}, \text { if } E_{i} \in(\pi-\varepsilon, 2 \pi+\varepsilon),
\end{array}\right.
$$

where

$$
\begin{aligned}
& V_{1}=\frac{1}{2}\left(\left\|l_{1}-l_{d_{1}}\right\|^{2}+\left\|A_{1}-A_{d_{1}}\right\|^{2}\right) \\
& V_{2}=\frac{1}{2}\left(\left\|l_{2}-l_{d_{2}}\right\|^{2}+\left\|A_{2}-A_{d_{2}}\right\|^{2}\right) .
\end{aligned}
$$

Here, $\left(l_{d_{1}}, A_{d_{1}}\right)$ and $\left(l_{d_{2}}, A_{d_{2}}\right)$ specify the orbits in a two-satellite periodic formation and $\phi$ specifies the desired $\left(M_{1}-M_{2}\right)$ on these orbits.

We calculate the derivative of $V$ as

$$
\dot{V}=\dot{V}_{1}+\dot{V}_{2}+\sin \left(\frac{\Upsilon_{1}-\Upsilon_{2} \mp \phi}{2}\right)\left(\dot{\Upsilon}_{1}-\dot{\Upsilon}_{2}\right) \text {. }
$$

The choice of - or + depends on the value of $E_{i}$ as in the definition of $V$. From calculations performed in the single satellite case,

$$
\dot{V}_{i}=\left[\left(l_{i}-l_{d_{i}}\right) \times q_{i}+l_{i} \times\left(A_{i}-A_{d_{i}}\right)+\left(\left(A_{i}-A_{d_{i}}\right) \times p_{i}\right) \times q_{i}\right] \cdot u_{i},
$$

for $i=1,2$. Thus,

$$
\begin{aligned}
\dot{V}=[ & \left(l_{1}-l_{d_{1}}+\zeta_{1} \sin \left(\frac{\Upsilon_{1}-\Upsilon_{2} \mp \phi}{2}\right) l_{1}\right) \times q_{1} \\
& +l_{1} \times\left(A_{1}-A_{d_{1}}+\rho_{1} \sin \left(\frac{\Upsilon_{1}-\Upsilon_{2} \mp \phi}{2}\right) \hat{A}_{1}\right) \\
& \left.+\left(\left(A_{1}-A_{d_{1}}+\rho_{1} \sin \left(\frac{\Upsilon_{1}-\Upsilon_{2} \mp \phi}{2}\right) \hat{A}_{1}\right) \times p_{1}\right) \times q_{1}\right] \cdot u_{1} \\
& +\left[\left(l_{2}-l_{d_{2}}+\zeta_{2} \sin \left(\frac{\Upsilon_{1}-\Upsilon_{2} \mp \phi}{2}\right) l_{2}\right) \times q_{2}\right. \\
& +l_{2} \times\left(A_{2}-A_{d_{2}}+\rho_{2} \sin \left(\frac{\Upsilon_{1}-\Upsilon_{2} \mp \phi}{2}\right) \hat{A}_{2}\right) \\
& \left.+\left(\left(A_{2}-A_{d_{2}}+\rho_{2} \sin \left(\frac{\Upsilon_{1}-\Upsilon_{2} \mp \phi}{2}\right) \hat{A}_{2}\right) \times p_{2}\right) \times q_{2}\right] \cdot u_{2} .
\end{aligned}
$$

To obtain $\dot{V} \leq 0$, we let 


$$
\begin{aligned}
u_{1}=- & \sin ^{2}\left(E_{1}\right)\left[\left(l_{1}-l_{d_{1}}+\zeta_{1} \sin \left(\frac{\Upsilon_{1}-\Upsilon_{2} \mp \phi}{2}\right) l_{1}\right) \times q_{1}\right. \\
& +l_{1} \times\left(A_{1}-A_{d_{1}}+\rho_{1} \sin \left(\frac{\Upsilon_{1}-\Upsilon_{2} \mp \phi}{2}\right) \hat{A}_{1}\right) \\
& \left.+\left(\left(A_{1}-A_{d_{1}}+\rho_{1} \sin \left(\frac{\Upsilon_{1}-\Upsilon_{2} \mp \phi}{2}\right) \hat{A}_{1}\right) \times p_{1}\right) \times q_{1}\right] \\
u_{2}=- & \sin ^{2}\left(E_{2}\right)\left[\left(l_{2}-l_{d_{2}}+\zeta_{2} \sin \left(\frac{\Upsilon_{1}-\Upsilon_{2} \mp \phi}{2}\right) l_{2}\right) \times q_{2}\right. \\
& +l_{2} \times\left(A_{2}-A_{d_{2}}+\rho_{2} \sin \left(\frac{\Upsilon_{1}-\Upsilon_{2} \mp \phi}{2}\right) \hat{A}_{2}\right) \\
& \left.+\left(\left(A_{2}-A_{d_{2}}+\rho_{2} \sin \left(\frac{\Upsilon_{1}-\Upsilon_{2} \mp \phi}{2}\right) \hat{A}_{2}\right) \times p_{2}\right) \times q_{2}\right] .
\end{aligned}
$$

Note that the factors $\sin ^{2}\left(E_{i}\right)$ cancel the term $\sin \left(E_{i}\right)$ in the denominators of $\zeta_{i}$ and $\rho_{i}$. This results in a continuous control law that will be 0 when $E_{i}=0, \pi$.

Another issue is that when $e_{i}=0, E_{i}$ is not well defined. Thus, we should not allow $A_{d_{i}}=0$. Furthermore, we should choose our initial value of function $V$ such that the subset of the space $\Sigma_{e_{1}} \times \Sigma_{e_{2}}$, where $A_{i}=0$, will not be reached by the controlled dynamics.

Let $z=\left(q_{1}, p_{1}, q_{2}, p_{2}\right)$. We now proceed to find the initial condition

$$
z_{0}=\left(q_{1}(0), p_{1}(0), q_{2}(0), p_{2}(0)\right)
$$

for $z$, such that the set

$$
S_{M}=\left\{z \mid V(z) \leq V\left(z_{0}\right)\right\}
$$

is a compact subset of $\Sigma_{e_{1}} \times \Sigma_{e_{2}}-\left\{z \mid A_{1}=0\right.$ or $\left.A_{2}=0\right\}$. This is a necessary step because we want to apply LaSalle's invariance principle to prove our main result.

\section{Lemma 4:}

Let

$$
c<\min \left\{c_{1}, c_{2}\right\}
$$

where

$$
c_{i}=\min \left\{\frac{1}{2}\left\|A_{d_{i}}\right\|^{2}, \frac{1}{2}\left\|l_{d_{i}}\right\|^{2}, \frac{1}{2}\left(\mu-\| A_{d_{i}} \mid\right)^{2}\right\}
$$

for $i=1,2$. Then the set

$$
S_{M}=\{z \mid V(z) \leq c\}
$$

is a compact subset of $\Sigma_{e_{1}} \times \Sigma_{e_{2}}-\left\{z \mid A_{1}=0\right.$ or $\left.A_{2}=0\right\}$.

\section{Proof:}

The first observation is that the set

$$
S_{1}=\left\{\left(q_{1}, p_{1}\right) \mid V\left(q_{1}, p_{1}\right) \leq c^{*}, c^{*}<c_{1}\right\}
$$


is a subset of $\Sigma_{e_{1}}$, that is, $S_{1} \cap \Sigma_{e_{1}}=S_{1}$. In fact, $c_{1}$ is the supremum of $V_{1}\left(q_{1}, p_{1}\right)$ on the set $\Sigma_{e_{1}}-\left\{\left(q_{1}, p_{1}\right) \mid A_{1}=0\right\}$. To see this, we solve a constrained maximization problem as follows:

$$
\sup \left\{\frac{1}{2}\left\|l_{1}-l_{d_{1}}\right\|^{2}+\left\|A_{1}-A_{d_{1}}\right\|^{2}\right\}
$$

under the constraints

$$
A_{1} \cdot l_{1}=0, l_{1} \neq 0, A_{1} \neq 0, \text { and }\left\|A_{1}\right\|<\mu .
$$

First, we need to calculate the supremum of the unconstrained maximization problem. It is easy to see that this value is infinite. Then, we need to calculate the minimum value subject to $l_{1}=0$. The result is $\frac{1}{2}\left\|l_{d}\right\|^{2}$, achieved when $A=A_{d}$. Similarly, the minimum subject to $A_{1}=0$ is $\frac{1}{2}\left\|A_{d}\right\|^{2}$. We should also calculate the minimum value subject to $\|A\|=\mu$. By applying the Lagrange multiplier method, we found this value to be $\frac{1}{2}\left(\mu-\left\|A_{d}\right\|\right)^{2}$. Thus,

$$
c_{1}=\min \left\{\frac{1}{2}\left\|A_{d_{1}}\right\|^{2}, \frac{1}{2}\left\|l_{d_{1}}\right\|^{2}, \frac{1}{2}\left(\mu-\left\|A_{d_{1}}\right\|\right)^{2}\right\}
$$

is the supremum of $V_{1}$ on the set $\Sigma_{e_{1}}-\left\{\left(q_{1}, p_{1}\right) \mid A_{1}=0\right\}$. Thus, we have proved that $S_{1} \subset \Sigma_{e_{1}}-\left\{\left(q_{1}, p_{1}\right) \mid A_{1}=0\right\}$.

Another observation is that $\pi\left(S_{1}\right)$ is a compact subset of $D_{1}$. Thus, by Corollary 2, $S_{1}$ is a compact subset of $\Sigma_{e_{1}}$.

We can make the same arguments for the case when $i=2$ to prove that $S_{2}$ is a compact subset of $\Sigma_{e_{2}}-\left\{\left(q_{2}, p_{2}\right) \mid A_{2}=0\right\}$. Hence, by letting $c<\min \left\{c_{1}, c_{2}\right\}$, it is true that

$$
S_{M} \subset S_{1} \times S_{2} \subset \Sigma_{e_{1}} \times \Sigma_{e_{2}}-\left\{z \mid A_{1}=0 \text { or } A_{2}=0\right\} .
$$

Thus, $S_{M}$ is a compact subset of $\Sigma_{e_{1}} \times \Sigma_{e_{2}}-\left\{z \mid A_{1}=0\right.$ or $\left.A_{2}=0\right\}$.

We can now apply LaSalle's invariance principle to show that the trajectory of the closed loop system, starting within $S_{M}$, converges to the maximal invariant subset of $S_{M}$, where $u(t)=0$ is satisfied for all $t$.

\section{Proposition 5:}

With $V, c$, and $u_{i}$ given, as in (24), (31), and (29), the trajectory starting from the point $\left(q_{10}, p_{10}, q_{20}, p_{20}\right)$ that satisfies

$$
V\left(q_{10}, p_{10}, q_{20}, p_{20}\right) \leq c
$$

will converge to the set for which

$$
\begin{aligned}
l_{i} & =l_{d_{i}} \\
A_{i} & =A_{d_{i}} \\
M_{1}-M_{2} & =\phi
\end{aligned}
$$

are satisfied for $i=1,2$.

Proof:

To calculate the invariant set, let $u_{1}=0$. When $\sin \left(E_{1}\right)=0$, we obtain 


$$
\begin{aligned}
& \left(l_{1}-l_{d_{1}}+\zeta_{1} \sin \left(\frac{\Upsilon_{1}-\Upsilon_{2} \mp \phi}{2}\right) l_{1}\right) \times q_{1} \\
& \quad+l_{1} \times\left(A_{1}-A_{d_{1}}+\rho_{1} \sin \left(\frac{\Upsilon_{1}-\Upsilon_{2} \mp \phi}{2}\right) \hat{A}_{1}\right) \\
& \quad+\left(\left(A_{1}-A_{d_{1}}+\rho_{1} \sin \left(\frac{\Upsilon_{1}-\Upsilon_{2} \mp \phi}{2}\right) \hat{A}_{1}\right) \times p_{1}\right) \times q_{1} \\
& \quad=0 .
\end{aligned}
$$

By taking inner products on both sides with $q_{1}(t)$ we obtain

$$
\left(l_{1} \times\left(A_{1}-A_{d_{1}}+\rho_{1} \sin \left(\frac{\Upsilon_{1}-\Upsilon_{2} \mp \phi}{2}\right) \hat{A}_{1}\right)\right) \cdot q_{1}=0 .
$$

This is equivalent to

$$
\left(l_{1} \times q_{1}(t)\right) \cdot\left(A_{1}-A_{d_{1}}+\rho_{1} \sin \left(\frac{\Upsilon_{1}-\Upsilon_{2} \mp \phi}{2}\right) \hat{A}_{1}\right)=0 .
$$

Let

$$
B=A_{1}-A_{d_{1}}+\rho_{1} \sin \left(\frac{\Upsilon_{1}-\Upsilon_{2} \mp \phi}{2}\right) \hat{A}_{1},
$$

then Equation (43) means that $B$ is perpendicular to the vector $l_{1} \times q_{1}(t)$. We see that the vector $B$ should stay in the plane spanned by $l_{1}$ and $q_{1}(t)$, as shown in Figure 1. However, from the assumption that $u_{1}(t)=0$, we know that $A_{1}$ and $l_{1}$ are constant vectors. The time varying vector $B$ will sweep a line segment passing the fixed point $\left(A_{1}-A_{d_{1}}\right)$. The direction of this line segment is aligned with $\hat{A}_{1}$. Thus, vector $B$ will be the intersection of the $\left(l_{1}, q_{1}\right)$ plane and this line segment. Because $q_{1}(t)$ is sweeping the orbital plane, the $\left(l_{1}, q_{1}\right)$ plane is identical at $t$ and $t+k T_{1}$, where $k$ is an integer and $T_{1}$ is the period of the first satellite. Since the line segment is not changed, the intersection points in these cases must be identical. Thus, we must have

$$
B(t)=B\left(t+k T_{1}\right) \text {. }
$$

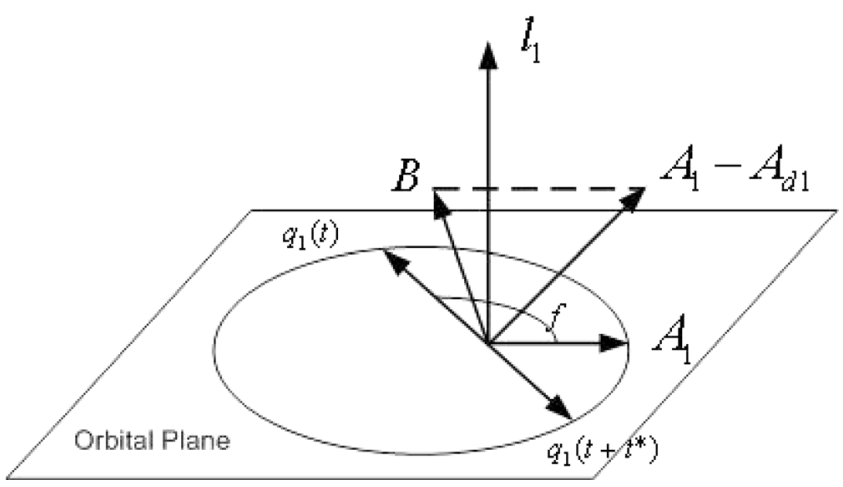

FIGURE 1. The relationship between $l_{1}, q_{1}, A_{1}-A_{d_{1}}$, and $B$ (see Proposition 5). 
Without lost of generality, suppose at time $t, E_{1}(t), E_{2}(t) \in(-\varepsilon, \pi+\varepsilon)$. Then, (45) requires that

$$
\begin{aligned}
& \rho_{1}\left(E_{1}(t)\right) \sin \left(\frac{\Upsilon_{1}(t)-\Upsilon_{2}(t)-\phi}{2}\right) \\
& \quad=\rho_{1}\left(E_{1}\left(t+k T_{1}\right)\right) \sin \left(\frac{\Upsilon_{1}\left(t+k T_{1}\right)-\Upsilon_{2}\left(t+k T_{1}\right)-\phi}{2}\right) .
\end{aligned}
$$

Let $k=1$ in Equation (46), because $\rho_{1}\left(E_{1}(t)\right)=\rho_{1}\left(E_{1}\left(t+T_{1}\right)\right)$, the first observation we make is that the two satellites must have the same period. In fact, suppose that at time $t_{0}$ Equation (46) is satisfied. Then, at time $t_{0}+T_{1}$, since $E_{1}\left(t_{0}\right)=E_{1}\left(t_{0}+T_{1}\right)$, $\Upsilon_{1}\left(t_{0}\right)=\Upsilon_{1}\left(t_{0}+T_{1}\right)$, and all the angles (anomalies) are in the interval $[0,2 \pi)$, we must have $\Upsilon_{2}\left(t_{0}\right)=\Upsilon_{2}\left(t_{0}+T_{1}\right)$. However,

$$
\Upsilon_{2}\left(t_{0}\right)-\Upsilon_{2}\left(t_{0}+T_{1}\right)=-\left(\frac{a_{2}}{a_{d}}\right)^{3 / 2}\left(M_{2}\left(t_{0}+T_{1}\right)-M_{2}\left(t_{0}\right)\right)
$$

Then $\Upsilon_{2}\left(t_{0}\right)=\Upsilon_{2}\left(t_{0}+T_{1}\right)$ is satisfied only if $M_{2}\left(t_{0}+T_{1}\right)=M_{2}\left(t_{0}\right)$. Thus, we have $T_{1}=k_{1} T_{2}$, where $k_{1}$ is a positive integer. Remember, we can apply the same argument to the second satellite to obtain $T_{2}=k_{1} T_{1}$. Thus, we must have $T_{1}=T_{2}$. Hence, on the invariant set, we have proved that $a_{1}=a_{2}$.

On the other hand, for a specific time $t$, we know that there exists $t^{*} \in\left[0, T_{1}\right)$ such that

$$
\pi+f_{1}(t)=f_{1}\left(t+t^{*}\right)
$$

where $f_{1}$ is the true anomaly of the first satellite. The value of $t^{*}$ depends on $t$. The plane spanned by $\left(l_{1}, q_{1}\right)$ at time $t$ is also identical to the plane spanned by $\left(l_{1}, q_{1}\right)$ at time $t+t^{*}$. Thus, we must have

$$
B(t)=B\left(t+t^{*}\right)
$$

which requires that

$$
\begin{aligned}
& \rho_{1}\left(E_{1}(t)\right) \sin \left(\frac{\Upsilon_{1}(t)-\Upsilon_{2}(t)-\phi}{2}\right) \\
& \quad=\rho_{1}\left(E_{1}\left(t+t^{*}\right)\right) \sin \left(\frac{\Upsilon_{1}\left(t+t^{*}\right)-\Upsilon_{2}\left(t+t^{*}\right)+\phi}{2}\right) .
\end{aligned}
$$

Furthermore, $a_{1}=a_{2}$ implies that $M_{1}(t)-M_{2}(t)=M_{1}\left(t+t^{*}\right)-M_{2}\left(t+t^{*}\right)$, and one can verify that

$$
\sin \left(\frac{\Upsilon_{1}(t)-\Upsilon_{2}(t)-\phi}{2}\right)=-\sin \left(\frac{\Upsilon_{1}\left(t+t^{*}\right)-\Upsilon_{2}\left(t+t^{*}\right)+\phi}{2}\right) .
$$

For (50) to be satisfied, one possibility is that

$$
\sin \left(\frac{\Upsilon_{1}(t)-\Upsilon_{2}(t)-\phi}{2}\right)=0
$$

Another possibility is that

$$
\rho_{1}\left(E_{1}(t)\right)=-\rho_{1}\left(E_{1}\left(t+t^{*}\right)\right) .
$$

By definition of $\rho_{1}$, one can verify that (53) can only be satisfied when $t$ takes value from a set of measure 0 . Thus, for (50) to be satisfied, (52) must be true. Because of 
(52), the time varying parts in Equation (41) vanish. We can make the same argument as in the proof for the single satellite case ${ }^{3}$ to show that

$$
\begin{gathered}
l_{1}=l_{d_{1}} \\
A_{1}=A_{d_{1}} .
\end{gathered}
$$

We can apply similar arguments for the second satellite. Thus, we have

$$
\begin{aligned}
l_{i} & =l_{d_{i}} \\
A_{i} & =A_{d_{i}} \\
\Upsilon_{1}-\Upsilon_{2} & = \pm \phi
\end{aligned}
$$

for $i=1,2$. By definition of $\Upsilon_{1}$ and $\Upsilon_{2}$ in Equation (18), we have

$$
\left(\frac{a_{1}}{a_{d}}\right)^{3 / 2} M_{1}-\left(\frac{a_{2}}{a_{d}}\right)^{3 / 2} M_{2}=\phi .
$$

However, we already know $a_{1}=a_{2}=a_{d}$, so we conclude that

$$
M_{1}-M_{2}=\phi \text {. }
$$

This proposition can be easily extended to $m$ satellites where $m>2$. Let the $m$ th satellite be the leader of the formation. Let

$$
V=\sum_{i}^{m}\left(V_{i}+4 \sin \left(\frac{\Upsilon_{1}-\Upsilon_{m} \mp \phi_{i}}{4}\right)^{2}\right),
$$

where $i=1,2,3, \ldots, m, \phi_{m}=0$, and

$$
V_{i}=\frac{1}{2}\left\|l_{i}-l_{d_{i}}\right\|^{2}+\left\|A_{i}-A_{d_{i}}\right\|^{2} .
$$

It is easy to see that the control for the $i$ th satellite with $i \leq m-1$ is the same as the control for the $(i, m)$ pair of satellites. The initial value of $V$ should be less than the minimum value of $c_{i}$ for $i=1,2, \ldots, m-1$. Then the solution will converge to the set where

$$
\begin{aligned}
l_{i} & =l_{d_{i}} \\
A_{i} & =A_{d_{i}} \\
M_{i}-M_{n} & =\phi_{i}
\end{aligned}
$$

are satisfied for $i=1,2, \ldots, m$.

\section{TRANSFER OF SATELLITE FORMATIONS TO CIRCULAR ORBITS}

Formations on circular orbits are of great importance. ${ }^{6}$ If the destination of at least one of the satellites in a formation is circular, there is difficulty in determining the mean anomaly. Since the Laplace vector $A$ vanishes, the perigee point cannot be determined. In this case, the position of a satellite on the circular orbit is usually described by using the argument of latitude, defined as the angle $\theta$ between the line 
of ascending node and the position vector $q$. It is easy to see that on an elliptic orbit, this angle is

$$
\theta=\omega+f
$$

where $\omega$ is the argument of perigee and $f$ is the true anomaly. On a circular orbit, we have

$$
\theta=n(t-\tau),
$$

where $\tau$ is the time when the satellite passes the ascending node for the first time. As can be seen, $\theta$ serves as the mean anomaly for circular orbit.

Although $\theta$ and $\dot{\theta}$ can be easily determined, as discussed in the APPENDIX, they are not suitable for our Lyapunov function based control laws because $\theta$ is not affine in time on an elliptic orbit. Thus, we use the angle $M_{\theta}$, which is the mean anomaly measured from the line of node. $M_{\theta}$ can be determined from $\theta$, but if the orbit is close to circular, we can use $\theta$ as an approximation for $M_{\theta}$. Our analysis in the APPENDIX shows that the derivative of $M_{\theta}$ is

$$
\begin{aligned}
\dot{M}_{\theta}=n & -\frac{r_{\omega}^{2}}{a^{2} \sqrt{1-e^{2}}} \frac{\cos (I)}{h \sin (I)}\left(\frac{\partial l}{\partial p}\right)^{T} \hat{n} \cdot u \\
& -\left(\sin (E)-\sin \left(E_{\omega}\right)+\frac{r \sin (E)-r_{\omega} \sin \left(E_{\omega}\right)}{a\left(1-e^{2}\right)}\right) \frac{1}{\mu}\left(\frac{\partial A}{\partial p}\right)^{T} \hat{A} \cdot u \\
& +\frac{r^{2}-r_{\omega}^{2}}{\mu a^{2} e \sqrt{1-e^{2}}}\left(\left(\frac{\partial A}{\partial p}\right)^{T} \hat{A}_{\perp} \cdot u\right) .
\end{aligned}
$$

Here, $\omega$ is the argument of perigee, $E$ and $E_{\omega}$ are the eccentric anomalies corresponding to $\theta-\omega$ and $\omega, r_{\omega}$ denotes the distance between the ascending node and the gravitational center. We let $\hat{n}=\left(l_{x}^{2}+l_{y}^{2}\right)^{-1 / 2}\left[-l_{y}, l_{x}, 0\right]^{T}$, where $\hat{n}$ is the unit vector pointing to the ascending node. Let $\hat{A}_{\perp}$ be the unit vector perpendicular to $\hat{A}$ and $l$ forming an acute angle with $\hat{n}$.

We now study the case when we want to set up a formation in which all the members are on circular orbits with the same radius. According to the previous discussion under PERIODIC Formation, this formation is a periodic formation. Let $\tau_{i}$ be the time when the $i$ th satellite passes the line of ascending node for the first time. Similar to the perigee passage time in our elliptic orbit case, $\tau_{i}$ is a constant of the free motion for satellite $i$. Hence, by specifying the differences $\tau_{i}-\tau_{j}$ or $\theta_{i}-\theta_{j}$ between satellites, a periodic formation on circular orbits can be uniquely determined.

Similar to the elliptic orbit case, we introduce a variable $\psi_{i}$, which is defined as

$$
\psi_{i}=\left\{\begin{array}{l}
\left(\frac{a_{i}}{a_{d}}\right)^{3 / 2} M_{\theta_{i}}, \text { if } \theta_{i} \in(-\varepsilon, \pi+\varepsilon) \\
\left(\frac{a_{i}}{a_{d}}\right)^{3 / 2}\left(2 \pi-M_{\theta_{i}}\right), \text { if } \theta_{i} \in(\pi-\varepsilon, 2 \pi+\varepsilon)
\end{array}\right.
$$

for $i=1,2$, where $a_{d}$ is the common length of the semimajor axes (or radius) for the destination orbits. Here, the reasons for using two charts are the same as in the case of elliptic orbits.

Our calculations in the APPENDIX show that the derivative of $\psi$ is 


$$
\dot{\psi}=\sqrt{\frac{\mu}{a_{d}^{3}}}+\left(\frac{\partial l}{\partial p}\right)^{T}(\lambda \hat{n}+\kappa l) \cdot u+\left(\frac{\partial A}{\partial p}\right)^{T}\left(\sigma \hat{A}+\varrho \hat{A}_{\perp}\right) \cdot u
$$

where for $\theta \in(-\varepsilon, \pi+\varepsilon)$,

$$
\begin{aligned}
\lambda & =-\left(\frac{a}{a_{d}}\right)^{3 / 2} \frac{r_{\omega}^{2}}{a^{2} \sqrt{1-e^{2}}} \frac{\cos (I)}{h \sin (I)} \approx-\left(\frac{a}{a_{d}}\right)^{3 / 2} \frac{r_{\omega}^{2}}{a} \frac{\cos (I)}{h \sin ^{2}(I)} \\
\kappa & =\frac{3}{\mu a\left(1-e^{2}\right)} \psi \\
\sigma & =\frac{3 e}{\mu\left(1-e^{2}\right)} \psi-\frac{1}{\mu}\left(\frac{a}{a_{d}}\right)^{3 / 2}\left(\sin (E)-\sin \left(E_{\omega}\right)+\frac{r \sin (E)-r_{\omega} \sin \left(E_{\omega}\right)}{a\left(1-e^{2}\right)}\right) \\
& \approx-\frac{1}{\mu}\left(\frac{a}{a_{d}}\right)^{3 / 2}\left(\sin (E)-\sin \left(E_{\omega}\right)+\frac{r \sin (E)-r_{\omega} \sin \left(E_{\omega}\right)}{a\left(1-e^{2}\right)}\right) \\
\varrho & =\left(\frac{a}{a_{d}}\right)^{3 / 2} \frac{r^{2}-r_{\omega}^{2}}{\mu a^{2} e \sqrt{1-e^{2}}} \approx-\left(\frac{a}{a_{d}}\right)^{3 / 2} \frac{2\left(\cos (E)-\cos \left(E_{\omega}\right)\right)}{\mu},
\end{aligned}
$$

and for $\theta \in(\pi-\varepsilon, 2 \pi+\varepsilon)$,

$$
\begin{aligned}
\lambda & =\left(\frac{a}{a_{d}}\right)^{3 / 2} \frac{r_{\omega}^{2}}{a^{2} \sqrt{1-e^{2}}} \frac{\cos (I)}{h \sin (I)} \approx\left(\frac{a}{a_{d}}\right)^{3 / 2} \frac{r_{\omega}^{2}}{a^{2}} \frac{\cos (I)}{h \sin (I)} \\
\kappa & =\frac{3}{\mu a\left(1-e^{2}\right)} \psi \\
\sigma & =\frac{3 e}{\mu\left(1-e^{2}\right)} \psi+\frac{1}{\mu}\left(\frac{a}{a_{d}}\right)^{3 / 2}\left(\sin (E)-\sin \left(E_{\omega}\right)+\frac{r \sin (E)-r_{\omega} \sin \left(E_{\omega}\right)}{a\left(1-e^{2}\right)}\right) \\
& \approx \frac{1}{\mu}\left(\frac{a}{a_{d}}\right)^{3 / 2}\left(\sin (E)-\sin \left(E_{\omega}\right)+\frac{r \sin (E)-r_{\omega} \sin \left(E_{\omega}\right)}{a\left(1-e^{2}\right)}\right) \\
\varrho & =-\left(\frac{a}{a_{d}}\right)^{3 / 2} \frac{r^{2}-r_{\omega}^{2}}{\mu a^{2} e \sqrt{1-e^{2}}} \approx\left(\frac{a}{a_{d}}\right)^{3 / 2} \frac{2\left(\cos (E)-\cos \left(E_{\omega}\right)\right)}{\mu} .
\end{aligned}
$$

We use the symbol $\approx$ when the eccentricity of the orbits are very small. When the orbit is circular, $E$ and $E_{\omega}$ are not well defined, but we have $M_{\theta}=\theta$ and

$$
\dot{\theta}=n-\frac{\cos (I)}{h \sin (I)}\left(\frac{\partial l}{\partial p}\right)^{T} \hat{n} \cdot u .
$$

Thus, when $e=0$

$$
\begin{aligned}
\lambda & =\mp\left(\frac{a}{a_{d}}\right)^{3 / 2} \frac{\cos (I)}{h \sin (I)} \\
\kappa & =\frac{3}{\mu a\left(1-e^{2}\right)} \psi \\
\sigma & =0 \\
\varrho & =0 .
\end{aligned}
$$


This result agrees with the limits of (66) and (67) as $e \rightarrow 0$.

Mimicking what was done in the elliptic orbit case, we design a Lyapunov function on the phase space of two satellites as follows:

$$
V=\left\{\begin{array}{l}
V_{1}+V_{2}+4 \sin \left(\frac{\psi_{1}-\psi_{2}-\phi}{4}\right)^{2}, \text { if } \theta_{i} \in(-\varepsilon, \pi+\varepsilon) \\
V_{1}+V_{2}+4 \sin \left(\frac{\psi_{1}-\psi_{2}+\phi}{4}\right)^{2}, \text { if } \theta_{i} \in(\pi-\varepsilon, 2 \pi+\varepsilon)
\end{array}\right.
$$

where

$$
\begin{aligned}
& V_{1}=\frac{1}{2}\left(\left\|l_{1}-l_{d_{1}}\right\|^{2}+\left\|A_{1}\right\|^{2}\right) \\
& V_{2}=\frac{1}{2}\left(\left\|l_{2}-l_{d_{2}}\right\|^{2}+\left\|A_{2}\right\|^{2}\right) .
\end{aligned}
$$

The derivative of this function has similar form to Equation (28). The control law $u$ such that $\dot{V} \leq 0$ can be chosen as

$$
\begin{aligned}
u_{1}=- & {\left[\left(l_{1}-l_{d_{1}}+\sin \left(\frac{\psi_{1}-\psi_{2} \mp \phi}{2}\right)\left(\lambda_{1} \hat{n}_{1}+\kappa_{1} l_{1}\right)\right) \times q_{1}\right.} \\
& +l_{1} \times\left(A_{1}+\sin \left(\frac{\psi_{1}-\psi_{2} \mp \phi}{2}\right)\left(\sigma_{1} \hat{A}_{1}+\varrho_{1} \hat{A}_{1 \perp}\right)\right) \\
& \left.+\left(\left(A_{1}+\sin \left(\frac{\psi_{1}-\psi_{2} \mp \phi}{2}\right)\left(\sigma_{1} \hat{A}_{1}+\varrho_{1} \hat{A}_{1 \perp}\right)\right) \times p_{1}\right) \times q_{1}\right] \\
u_{2}=- & {\left[\left(l_{2}-l_{d_{2}}+\sin \left(\frac{\psi_{1}-\psi_{2} \mp \phi}{2}\right)\left(\lambda_{2} \hat{n}_{2}+\kappa_{2} l_{2}\right)\right) \times q_{2}\right.} \\
& +l_{2} \times\left(A_{2}-\sin \left(\frac{\psi_{1}-\psi_{2} \mp \phi}{2}\right)\left(\sigma_{2} \hat{A}_{2}+\varrho_{2} \hat{A}_{2 \perp}\right)\right) \\
& \left.+\left(\left(A_{2}-\sin \left(\frac{\psi_{1}-\psi_{2} \mp \phi}{2}\right)\left(\sigma_{2} \hat{A}_{2}+\varrho_{2} \hat{A}_{2 \perp}\right)\right) \times p_{2}\right) \times q_{2}\right] .
\end{aligned}
$$

Based on Lemma 4, the following lemma is obvious.

Lemma 6:

Let

$$
c<\min \left\{c_{1}, c_{2}\right\}
$$

where

$$
c_{i}=\min \left\{\frac{1}{2}\left\|l_{d_{i}}\right\|^{2}, \frac{1}{2} \mu^{2}\right\}
$$

for $i=1,2$. Then the set

$$
S_{M}=\{z \mid V(z) \leq c\}
$$

is a compact subset of $\Sigma_{e_{1}} \times \Sigma_{e_{2}}$. 
Hence we prove that, starting within the set $S_{M}$, the controlled dynamics will converge to the maximal invariant set where formation relationships are obtained.

\section{Proposition 7:}

With $V, c$, and $u_{i}$ given as in (70), (73), and (72), the trajectory starting from the point $\left(q_{10}, p_{10}, q_{20}, p_{20}\right)$ that satisfies

$$
V\left(q_{10}, p_{10}, q_{20}, p_{20}\right) \leq c
$$

will converge to the set for which

$$
\begin{aligned}
l_{i} & =l_{d_{i}} \\
A_{i} & =0 \\
\theta_{1}-\theta_{2} & =\phi
\end{aligned}
$$

are satisfied for $i=1,2$.

\section{Proof:}

Let $u_{1}=0$, and take the inner product with $q_{1}$ on both sides of the equation to obtain

$$
\left(l_{1} \times B_{1}\right) \cdot q_{1}=0 \text {, }
$$

where

$$
B_{1}=A_{1}+\sin \left(\frac{\psi_{1}-\psi_{2} \mp \phi}{2}\right)\left(\sigma_{1} \hat{A}_{1}+\varrho_{1} \hat{A}_{1 \perp}\right) .
$$

At the time $t_{0}$ when $E_{1}\left(t_{0}\right)=E_{\omega_{1}}$, since $M_{\theta_{1}}=0$ and $r_{1}=r_{\omega_{1}}$, we have

$$
\varrho_{1}\left(t_{0}\right)=0 \text { and } \sigma_{1}\left(t_{0}\right)=0
$$

hence

$$
B_{1}\left(t_{0}\right)=A_{1}
$$

On the other hand,

$$
\left(q_{1} \times l_{1}\right) \cdot B_{1}=0
$$

tells us that $B_{1}$ lies in the plane spanned by $l_{1}$ and $q_{1}$. Thus, when $q_{1}\left(t_{0}\right)$ is not aligned with $A_{1}, B_{1}\left(t_{0}\right)$ must vanish. This implies that

$$
A_{1}=0 \text {. }
$$

However, it is also possible that $q_{1}\left(t_{0}\right)$ is aligned with $A_{1}$. In this case we have

$$
E_{\omega_{1}}=0 \text { and } \omega_{1}=0 \text {. }
$$

Therefore, for all time $t$,

$$
\begin{aligned}
& \varrho_{1}(t)= \pm\left(\frac{a_{1}}{a_{d}}\right)^{3 / 2} \frac{r_{1}^{2}-r_{\omega_{1}}^{2}}{\mu a_{1}^{2} e_{1} \sqrt{1-e_{1}^{2}}} \\
& \sigma_{1}(t)=\frac{3 e_{1}}{\mu\left(1-e_{1}^{2}\right)} \psi_{1}-\frac{1}{\mu}\left(\frac{a_{1}}{a_{d}}\right)^{3 / 2} \sin \left(E_{1}\right)\left(1+\frac{r_{1}}{a_{1}\left(1-e_{1}^{2}\right)}\right) .
\end{aligned}
$$

Thus, 


$$
B_{1}(t)=A_{1}+\sin \left(\frac{\psi_{1}-\psi_{2} \mp \phi}{2}\right)\left(\sigma_{1} \hat{A}_{1}+\varrho_{1} \hat{A}_{1 \perp}\right),
$$

and $B_{1}(t)$ has to be aligned with $q_{1}(t)$ for all time $t$. If we let $T_{1}$ be the orbital period of the first satellite and $T_{2}$ be the orbital period of the second satellite, then after one period $T_{1}$ the first satellite will return to the same position, which implies that

$$
\begin{aligned}
A_{1} & +\sin \left(\frac{\Psi_{1}\left(t+T_{1}\right)-\psi_{2}\left(t+T_{1}\right) \mp \phi}{2}\right)\left(\sigma_{1}\left(t+T_{1}\right) \hat{A}_{1}+\varrho_{1}\left(t+T_{1}\right) \hat{A}_{1 \perp}\right) \\
= & A_{1}+\sin \left(\frac{\psi_{1}(t)-\psi_{2}(t) \mp \phi}{2}\right)\left(\sigma_{1}(t) \hat{A}_{1}+\varrho_{1}(t) \hat{A}_{1 \perp}\right) .
\end{aligned}
$$

Because $M_{\theta_{1}}(t)=M_{\theta_{1}}\left(t+T_{1}\right)$ and $E_{1}(t)=E_{1}\left(t+T_{1}\right)$, we know $\sigma_{1}\left(t+T_{1}\right)=\sigma_{1}(t)$ and $\varrho_{1}\left(t+T_{1}\right)=\varrho_{1}(t)$. Thus, the above equation results in

$$
\begin{aligned}
& \sin \left(\frac{\left(\frac{a_{1}}{a_{d}}\right)^{3 / 2} M_{\theta_{1}}(t)-\left(\frac{a_{2}}{a_{d}}\right)^{3 / 2} M_{\theta_{2}}(t)-\phi}{2}\right) \\
& =\sin \left(\frac{\left(\frac{a_{1}}{a_{d}}\right)^{3 / 2} M_{\theta_{1}}(t)-\left(\frac{a_{2}}{a_{d}}\right)^{3 / 2} M_{\theta_{2}}\left(t+T_{1}\right)-\phi}{2}\right) .
\end{aligned}
$$

Therefore, we must have

$$
M_{\theta_{2}}(t)=M_{\theta_{2}}\left(t+T_{1}\right) .
$$

Hence, $T_{1} / T_{2}$ is a positive integer. On the other hand, the above arguments can be applied to the second satellite. If $A_{2} \neq 0, T_{2} / T_{1}$ must be a positive integer. Hence, $T_{2}=T_{1}$, which implies that $a_{1}=a_{2}$.

Still assuming $a_{1}\left(t_{0}\right)$ is aligned with $A_{1}$, we know that by a property of elliptic orbits, after half period, $q_{1}\left(t_{0}+T_{1} / 2\right)$ is aligned with $A_{1}$ again, which means that

$$
\sin \left(\frac{\psi_{1}\left(t_{0}+T_{1} / 2\right)-\psi_{2}\left(t_{0}+T_{1} / 2\right) \mp \phi}{2}\right) \varrho_{1}\left(t_{0}+T_{1} / 2\right)=0 .
$$

According to the definition of $\psi$, one can verify that

$$
\sin \left(\frac{\psi_{1}\left(t+T_{1} / 2\right)-\psi_{2}\left(t+T_{1} / 2\right) \mp \phi}{2}\right)=-\sin \left(\frac{\psi_{1}(t)-\psi_{2}(t) \mp \phi}{2}\right) .
$$

Therefore, the first possibility is that

$$
\mathrm{s}_{1}\left(t_{0}+T_{1} / 2\right)=-\varrho_{1}\left(t_{0}\right)=0 .
$$

This further implies that $r_{1}\left(t_{0}+T_{1} / 2\right)=r_{1}\left(t_{0}\right)$. Because at time $t_{0}$ the satellite is at perigee and at time $t_{0}+T_{1} / 2$ the satellite is at apogee, we conclude that the orbit is circular; that is, $A_{1}=0$.

The second possibility for Equation (90) to hold is that

$$
\sin \left(\frac{\psi_{1}\left(t_{0}\right)-\psi_{2}\left(t_{0}\right) \mp \phi}{2}\right)=0 .
$$

However, because $T_{1}=T_{2}$, we must have 


$$
\psi_{1}(t)-\psi_{2}(t)=\psi_{1}\left(t_{0}\right)-\psi_{2}\left(t_{0}\right)
$$

which implies that

$$
\sin \left(\frac{\psi_{1}(t)-\psi_{2}(t) \mp \phi}{2}\right)=0
$$

One can make the same argument as in the proof of the single satellite case $\mathrm{e}^{3}$ to show that

$$
\begin{gathered}
l_{1}=l_{d_{1}} \\
A_{1}=0 .
\end{gathered}
$$

To this point, we have proved that our algorithm will cause either $A_{1}$ or $A_{2}$ to vanish. Without loss of generality, we proceed by assuming $A_{1}=0$. Then, from $u_{1}=0$, we get

$$
\left(l_{1}-l_{d_{1}}+\sin \left(\frac{\psi_{1}-\psi_{2} \mp \phi}{2}\right)\left(\lambda_{1} \hat{n}_{1}+\kappa_{1} l_{1}\right)\right) \times q_{1}=0 \text {. }
$$

Let

$$
D_{1}=l_{1}-l_{d_{1}}+\sin \left(\frac{\psi_{1}-\psi_{2} \mp \phi}{2}\right)\left(\lambda_{1} \hat{n}_{1}+\kappa_{1} l_{1}\right) .
$$

$D_{1}$ must be aligned with $q_{1}$ and also lies in the plane passing $\left(l_{1}-l_{d_{1}}\right)$ spanned by $l_{1}$ and $n_{1}$. Because the plane is fixed and $q_{1}$ is periodic in time, at time $t$ and time $\left(t+T_{1}\right)$, the intersection of $q_{1}$ and the plane must be the same. This tells us that $T_{1} / T_{2}$ is a positive integer. Now, if $A_{2}=0$, the above arguments can be repeated so that we conclude $T_{2} / T_{1}$ is also a positive integer. If $A_{2} \neq 0$, we can repeat the arguments following Equation (84) to conclude that $T_{2} / T_{1}$ is also a positive integer. Therefore, we conclude that $T_{1}=T_{2}$ and $a_{1}=a_{2}$.

According to a property of circular orbits, at times $t$ and $\left(t+T_{1} / 2\right)$, the intersection of $q_{1}$ and the plane spanned by $l_{1}$ and $n_{1}$ passing $l_{1}-l d_{1}$ should be the same, that is,

$$
D_{1}(t)=D_{1}\left(t+T_{1} / 2\right) \text {. }
$$

Thus,

$$
\begin{aligned}
& \sin \left(\frac{\Psi_{1}(t)-\Psi_{2}(t) \mp \phi}{2}\right)\left(\lambda_{1}(t) \hat{n}_{1}+\kappa_{1}(t) l_{1}\right) \\
& =\sin \left(\frac{\Psi_{1}\left(t+T_{1} / 2\right)-\psi_{2}\left(t+T_{1} / 2\right) \mp \phi}{2}\right)\left(\lambda_{1}\left(t+T_{1} / 2\right) \hat{n}_{1}+\kappa_{1}\left(t+T_{1} / 2\right) l_{1}\right) .
\end{aligned}
$$

Because of (91), Equation (100) is satisfied only if

$$
\sin \left(\frac{\psi_{1}(t)-\psi_{2}(t) \mp \phi}{2}\right)=0
$$

or

$$
\lambda_{1}(t) \hat{n}_{1}+\kappa_{1}(t) l_{1}=-\lambda_{1}\left(t+T_{1} / 2\right) \hat{n}_{1}-\kappa_{1}\left(t+T_{1} / 2\right) l_{1} .
$$

Equation (102) implies that for all time $t$,

$$
\kappa(t)=-\kappa\left(t+T_{1} / 2\right),
$$

which further implies that

$$
M_{\theta_{1}}(t)=\frac{\pi}{2}
$$


This is impossible. Thus, the only possibility is that

$$
\sin \left(\frac{\psi_{1}(t)-\psi_{2}(t) \mp \phi}{2}\right)=0
$$

Because of (105), the time varying parts in the equation $u_{1}=0$ vanish. We can make the same argument as in the proof for the single satellite case ${ }^{3}$ to show that

$$
\begin{gathered}
l_{1}=l_{d_{1}} \\
A_{1}=0 .
\end{gathered}
$$

Now let $u_{2}(t)=0$ and substitute Equation (105). We conclude that the second satellite will achieve

$$
\begin{aligned}
l_{2} & =l_{d_{2}} \\
A_{2} & =0 .
\end{aligned}
$$

Thus, we have

$$
\begin{aligned}
l_{i} & =l_{d_{i}} \\
A_{i} & =0 \\
\psi_{1}-\psi_{2} & = \pm \phi
\end{aligned}
$$

for $i=1,2$. By definition of $\psi_{1}$ and $\psi_{2}$ in (64), we have

$$
\left(\frac{a_{1}}{a_{d}}\right)^{3 / 2} M_{\theta_{1}}-\left(\frac{a_{2}}{a_{d}}\right)^{3 / 2} M_{\theta_{2}}=\phi .
$$

However, we already know $a_{1}=a_{2}=a_{d}$ and that the orbits are circular; thus, we conclude that

$$
\theta_{1}-\theta_{2}=\phi
$$

We mention that this algorithm is not limited to set up formations on circular orbits. With slight modification to the proof one can show the convergence result holds for elliptic orbits.

\section{SIMULATION RESULTS}

To verify our algorithms, a series of simulations were carried out using Matlab. First, we show a controlled transfer of two satellites from orbit $[a, e, i, \omega, \Omega]=$ $[20,0.1, \pi / 4, \pi / 2,0]$ with initial separation of mean anomaly $\pi / 90$ to the orbit $[a, e, i, \omega, \Omega]=[20,0.05, \pi / 3, \pi / 2,0]$ with final separation of mean anomaly $\pi / 18 \approx$ 0.175 . Only relative motion between the satellites is plotted in an inertial frame centered at one of the satellites. FIGURE 2 displays the desired relative motion between the satellites. FIGURE 3 displays the relative motion between the satellites using the proposed control algorithm. The final orbits for the two satellites are:

$$
\begin{aligned}
& {\left[a_{1}, e_{1}, I_{1}, \omega_{1}, \Omega_{1}\right]=[25.10,0.0495,1.0472,1.6231,0]} \\
& {\left[a_{2}, e_{2}, I_{2}, \omega_{2}, \Omega_{2}\right]=[25.12,0.0507,1.0472,1.6231,0]}
\end{aligned}
$$

and the final separation of mean anomaly is 0.168 . As can be seen, the desired orbit and separation are achieved with small error. We noticed that by adjusting the 


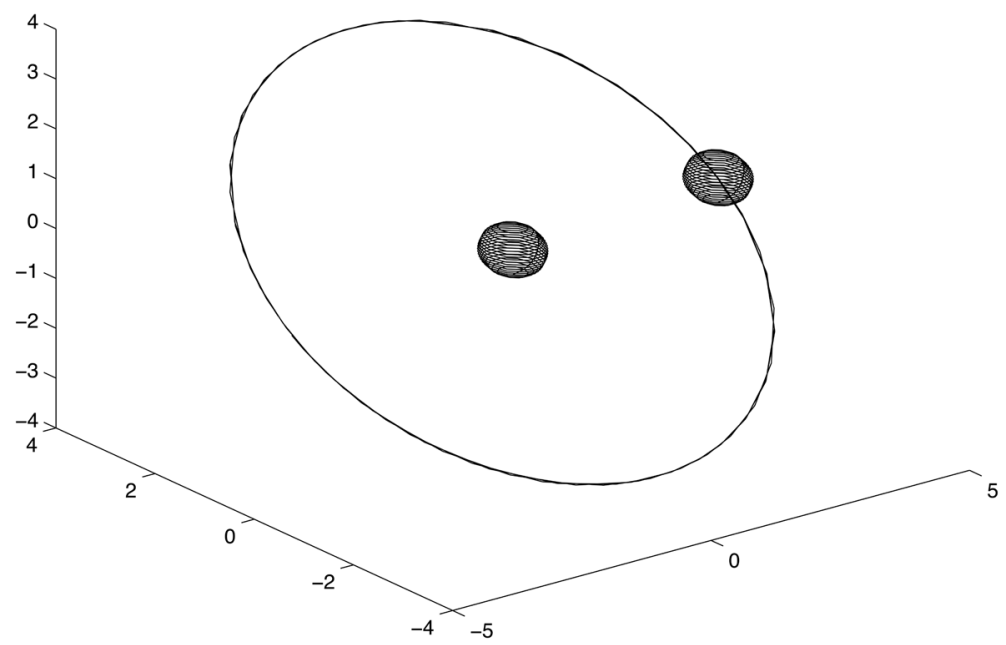

FIGURE 2. The desired final relative motion of two satellites (length unit, one tenth of Earth radius).

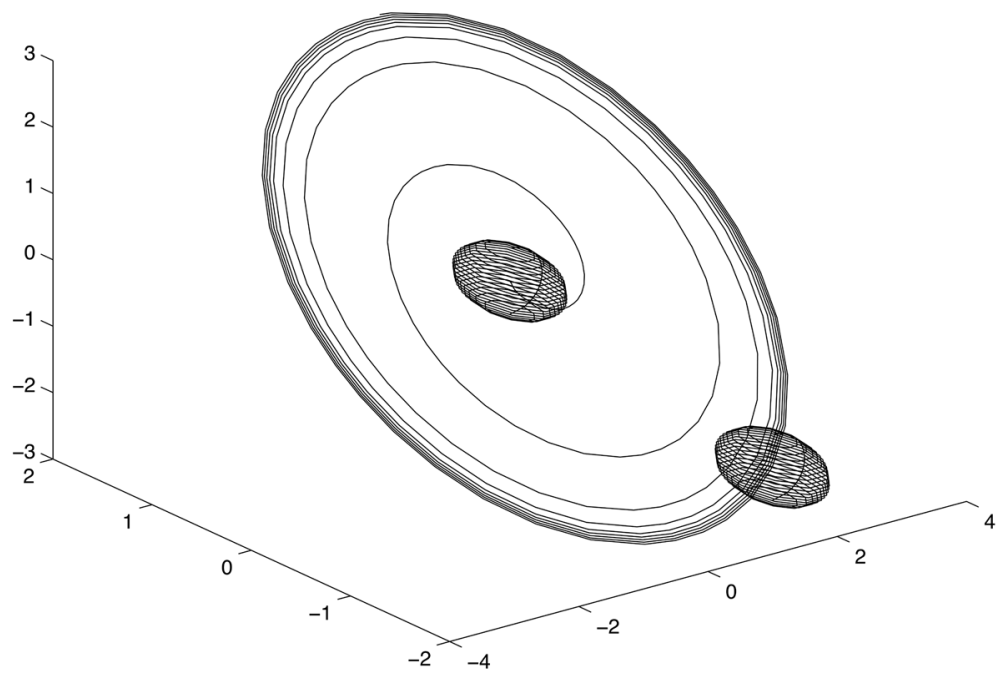

FIGURE 3. The relative motion achieved by our control law for elliptic orbits (length unit, one tenth of Earth radius). 
weight of the terms in our Lyapunov functions, we can control the distribution of errors among the orbital elements of final orbits.

Similar experiments were performed to set up a two satellite formation in circular orbits. Initially, the two satellites were on an elliptic orbit with $[a, e, i, \omega, \Omega]=$ $[20,0.1, \pi / 4, \pi / 2,0]$ and the separation of argument of latitude equal to $\pi / 90$. Their destination orbit was circular with $[a, e, i, \omega, \Omega]=[25,0, \pi / 3, \pi / 4]$ and separation $\pi / 18$. Using our algorithm, the final orbits obtained are:

$$
\begin{aligned}
& {\left[a_{1}, e_{1}, I_{1}, \Omega_{1}\right]=[25.082,0.0023,1.0472,0.7854]} \\
& {\left[a_{2}, e_{2}, I_{2}, \Omega_{2}\right]=[25.078,0.0022,1.0472,0.7854]}
\end{aligned}
$$

and the final separation equal to 0.174 . Again, the formation is achieved with small error.

\section{SUMMARY AND FUTURE DIRECTIONS}

In this paper we have proposed control algorithms that can be used to set up periodic formations of satellites on elliptic and circular orbits. The shape space formed by the angular momentum vectors and Laplace vectors is appropriate to describe satellite formations. The control laws we propose are based on a Lyapunov function on this shape space and proved to be convergent. We have not considered the effect of perturbations, such as the $J_{2}$ effect. This is currently being investigated.

\section{ACKNOWLEDGMENTS}

This research was supported in part by the National Aeronautics and Space Administration under NASA-GSFC Grant NAG5-10819, by the Air Force Office of Scientific Research under AFOSR Grant F49620-01-0415, by the Army Research Office under ODDR\&E MURI97 Program Grant DAAG55-97-1-0114 to the Center for Dynamics and Control of Smart Structures (through Harvard University), and under ODDR\&E MURI01 Program Grant DAAD19-01-1-0465 to the Center for Communicating Networked Control Systems (through Boston University).

\section{REFERENCES}

1. Schaub, H., S.R. Vadali, J.L. Junkins \& K.T. Alfriend. 2000. Spacecraft formation flying control using mean orbit elements. AAS J. Astronaut. Sci. 48(1): 69-87.

2. ZhANG, F. \& P.S. KRISHNAPRASAD. 2002. Formation dynamics under a class of control laws. In Proc. 2002 American Control Conference, Anchorage, Alaska. 1678-1685.

3. Chang, D.E., D. Chichka \& J.E. Marsden. 2002. Lyapunov-based transfer between elliptic Keplerian orbits. Discrete Contin. Dynam. Syst. B 2: 57-67.

4. Cushman R.H. \& L.M. Bates. 1997. Global Aspects of Classical Integrable Systems. Birkhauser Verlag, Switzerland.

5. Danby, J.M.A. 1962. Fundamentals of Celestial Mechanics. Macmillan, New York.

6. KRISHNAPRASAD, P.S. 2000. Relative equilibria and stability of rings of satellites. In Proc. 39th IEEE Conference on Decision and Control, Sydney, Australia. 1285-1288.

7. BuRns, J.A. 1976. Elementary derivation of the perturbation equations of celestial mechanics. Am. J. Phys. 44(10): 944-949.

8. VinTI, J.P. 1998. Orbital and Celestial Mechanics. AIAA. Reston, Virginia. 


\section{APPENDIX}

In this appendix we derive the perturbation equations for the orbital elements used in our control algorithm. The control forces are continuous functions of time. Hence, the derivatives are taken along the controlled dynamics of a satellite.

Starting with the eccentricity, we have

$$
\dot{e}(t)=\frac{A \cdot \dot{A}}{\mu\|A\|}=\frac{A}{\mu\|A\|} \cdot \frac{\partial A}{\partial p} u(t)=\frac{1}{\mu}\left(\frac{\partial A}{\partial p}\right)^{T} \hat{A} \cdot u(t),
$$

where $\hat{A}$ is the unit vector along the direction of $A$. We also have

$$
\dot{h}(t)=\frac{l \cdot l}{\|l\|}=\frac{1}{h}\left(\frac{\partial l}{\partial p}\right)^{T} l \cdot u(t)
$$

and

$$
\begin{aligned}
\dot{a}(t) & =\frac{1}{\mu\left(1-e^{2}\right)}(2 h \dot{h}+2 \mu a e \dot{e}) \\
& =\frac{2}{\mu\left(1-e^{2}\right)}\left(\frac{\partial l}{\partial p}\right)^{T} l \cdot u(t)+\frac{2 a e}{\mu\left(1-e^{2}\right)}\left(\frac{\partial A}{\partial p}\right)^{T} \hat{A} \cdot u(t) .
\end{aligned}
$$

We now derive the formulæ for $\dot{M}$. Since $r^{2}=q \cdot q$, we have $r \dot{r}=q \cdot p$. Hence

$$
\begin{aligned}
h^{2} & =l \cdot l=(q \times p) \cdot(q \times p) \\
& =(q \cdot q)(p \cdot p)-(q \cdot p)(q \cdot p)=r^{2} \mu\left(\frac{2}{r}-\frac{1}{a}\right)-r^{2} \dot{r}^{2} .
\end{aligned}
$$

On the other hand

$$
h^{2}=\mu a\left(1-e^{2}\right) \text {. }
$$

Thus,

$$
\dot{r}^{2}=-\frac{\mu a\left(1-e^{2}\right)}{r}+\mu\left(\frac{2}{r}-\frac{1}{a}\right) .
$$

After applying $r=a(1-e \cos (E))$, on simplification we obtain

$$
\dot{r}=\sqrt{\mu a} \frac{e \sin (E)}{r} .
$$

Taking derivatives on both sides of $r=a(1-e \cos (E))$,

$$
\dot{r}=\dot{a}(1-e \cos (E))+a(\dot{e} \cos (E)-e \sin (E) \dot{E}) .
$$

Thus,

$$
\begin{aligned}
\dot{E} & =\frac{\dot{r}}{a e \sin (E)}-\frac{r}{a^{2} e \sin (E)} \dot{a}+\frac{a \cos (E)}{a e \sin (E)} \dot{e} \\
& =\sqrt{\frac{\mu}{a}} \frac{1}{r}+\frac{\cos (E)}{e \sin (E)} \dot{e}-\frac{r}{a^{2} e \sin (E)} \dot{a} .
\end{aligned}
$$

Taking derivatives on both sides of $M=E-e \sin (E)$,

$$
\dot{M}=\dot{E}-\dot{e} \sin (E)-e \cos (E) \dot{E}=(1-e \cos (E)) \dot{E}-\dot{e} \sin (E) \text {. }
$$

Combining this equation with Equations (119), (113), and (111), we obtain 


$$
\begin{aligned}
\dot{M} & =(1-e \cos (E)) \sqrt{\frac{\mu}{a}} \frac{1}{r}+\left(\frac{\cos (E)(1-e \cos (E))}{e \sin (E)}-\sin (E)\right) \dot{e}-\frac{r(1-e \cos (E))}{a^{2} e \sin (E)} \dot{a} \\
& =\sqrt{\frac{\mu}{a^{3}}}+\frac{\cos (E)-e}{e \sin (E)} \dot{e}-\frac{(1-e \cos (E))^{2}}{a e \sin (E)} \dot{a} \\
& =n+\eta\left(\frac{\partial A}{\partial p}\right)^{T} \hat{A} \cdot u(t)+\xi\left(\frac{\partial l}{\partial o}\right)^{T} l \cdot u(t)
\end{aligned}
$$

where $n=\sqrt{\mu / a^{3}}$ and

$$
\begin{aligned}
\xi(l, A, E) & =-\frac{2(1-e \cos (E))^{2}}{\mu a e\left(1-e^{2}\right) \sin (E)} \\
\eta(l, A, E) & =\frac{\cos (E)-e}{\mu e \sin (E)}-\frac{2(1-e \cos (E))^{2}}{\mu\left(1-e^{2}\right) \sin (E)} .
\end{aligned}
$$

The inclination $I$ is defined as the angle between the $z$-axis and the angular momentum vector $l$. It can be determined from

$$
\cos (I)=\frac{l_{z}}{h} .
$$

Taking derivatives on both sides, we obtain

$$
\begin{aligned}
-\sin (I) \dot{I} & =-\frac{l_{z} l_{x} \dot{l}_{x}+l_{z} l_{y} \dot{l}_{y}-\left(l_{x}^{2}+l_{y}^{2}\right) \dot{l}_{z}}{h^{3}} \\
& =-\frac{\sin (I)}{h}[\cos (I) \sin (\Omega),-\cos (I) \cos (\Omega),-\sin (I)] \dot{i},
\end{aligned}
$$

because

$$
\cos (\Omega)=\frac{-l_{y}}{\sqrt{l_{x}^{2}+l_{y}^{2}}}=\frac{-l_{y}}{h \sin (I)} .
$$

Thus,

$$
\dot{I}=\frac{1}{h}[\cos (I) \sin (\Omega),-\cos (I) \cos (\Omega),-\sin (I)]\left(\frac{\partial l}{\partial p}\right) u .
$$

The argument of the ascending node $\Omega$ is defined as the angle between the $x$-axis and the ascending node. It can be determined from

$$
\tan (\Omega)=-\frac{l_{x}}{l_{y}}
$$

By taking derivatives on both sides of (127), we obtain

$$
\frac{1}{\cos ^{2}(\Omega)} \dot{\Omega}=-\frac{l_{y} l_{x}-l_{x} \dot{l}_{y}}{l_{y}^{2}}=-\frac{1}{l_{y}^{2}}\left[l_{y},-l_{x}, 0\right] \frac{\partial l}{\partial p} u .
$$

Let $\hat{n}=\left(l_{x}^{2}-l_{y}^{2}\right)^{-1 / 2}\left[-l_{y}, l_{x}, 0\right]^{T}$, then

$$
\dot{\Omega}=\frac{h \sin (I) \cos ^{2}(\Omega)}{l_{y}^{2}}\left(\frac{\partial l}{\partial p}\right)^{T} \hat{n} \cdot u=\frac{1}{h \sin (I)}\left(\frac{\partial l}{\partial p}\right)^{T} \hat{n} \cdot u .
$$

Note that $\hat{n}$ is the unit vector pointing to the ascending node. 
The argument of the perigee $\omega$ is defined as the angle between the vector $\hat{n}$ and $\hat{A}$. It can be determined from

$$
\cos (\omega)=\hat{n} \cdot \hat{A} .
$$

Taking derivatives on both sides,

$$
\dot{\omega}=-\frac{1}{\sin (\omega)}(\dot{\hat{n}} \cdot \hat{A}+\dot{\hat{A}} \cdot \hat{n}) \text {. }
$$

Because

$$
\dot{\hat{A}}=\frac{1}{\mu e}\left(I d-\hat{A} \hat{A}^{T}\right) \dot{A}
$$

and

$$
\dot{\hat{n}}=\frac{1}{h \sin (I)}\left(I d-\hat{n} \hat{n}^{T}\right) \dot{n},
$$

where $I d$ is the $3 \times 3$ identity matrix, one can verify that

$$
\dot{\hat{n}} \cdot \hat{A}=\dot{\Omega} \cos (I) \sin (\omega)
$$

and

$$
\dot{\hat{A}} \cdot \hat{n}=\frac{1}{\mu e}(\hat{n}-\cos (\omega) \hat{A}) \cdot \dot{A}=\frac{1}{\mu e} \sin (\omega) \hat{A}_{\perp} \cdot \dot{A}
$$

where $\hat{A}_{\perp}$ is the unit vector perpendicular to $\hat{A}$ and $l$ forming an acute angle with $\hat{n}$. The derivative of $\omega$ is

$$
\dot{\omega}=-\frac{\cos (I)}{h^{2} \sin ^{2}(I)}\left(\frac{\partial l}{\partial p}\right)^{T} \hat{n} \cdot u-\frac{1}{\mu e}\left(\frac{\partial A}{\partial p}\right)^{T} \hat{A}_{\perp} \cdot u .
$$

The longitude parameter $\theta$ is used instead of the true anomaly when the orbit is circular. To determine $\theta$, we have

$$
q \cdot \hat{n}=q_{x} \cos (\Omega)+q_{y} \sin (\Omega)=r \cos (\theta) .
$$

Hence,

$$
\cos (\theta)=\frac{x}{r} \cos (\Omega)+\frac{y}{r} \sin (\Omega) .
$$

On the other hand,

$$
-q \times \hat{n}=r \sin (\theta) \frac{l}{h} .
$$

This gives the following set of equations:

$$
\begin{aligned}
& \sin (\theta) \frac{l_{x}}{h}=\frac{z}{r} \sin (\Omega) \\
& \sin (\theta) \frac{l_{y}}{h}=-\frac{z}{r} \cos (\Omega) \\
& \sin (\theta) \frac{l_{z}}{h}=-\frac{x}{r} \sin (\Omega)+\frac{y}{r} \cos (\Omega) .
\end{aligned}
$$

Thus, $\theta$ can be uniquely determined by using Equation (138) and any one of (140).

We now calculate the derivative of $\theta$, which contains two parts. The first part is due to the orbital motion of the satellite and the second part is due to the perturbation of the line of ascending node; that is, 


$$
\dot{\theta}=\frac{h}{r^{2}}-\frac{\cos (I)}{h \sin (I)}\left(\frac{\partial l}{\partial p}\right)^{T} \hat{n} \cdot u .
$$

We now derive Equation (63) for $\dot{M}_{\theta}$. We start from the cosine relationship between the true anomaly $f$ and the eccentric anomaly $E$

$$
\cos (E)=\frac{e+\cos (f)}{1+e \cos (f)}
$$

Taking derivatives on both sides yields

$$
-\sin (E) \dot{E}=\frac{\left(e^{2}-1\right) \sin (f)}{(1+e \cos (f))^{2}} \dot{f}+\frac{\sin ^{2}(f)}{(1+e \cos (f))^{2}} \dot{e} .
$$

Because

$$
\sin (E)=\frac{\sqrt{1-e^{2}} \sin (f)}{1+e \cos (f)}
$$

we have

$$
\dot{E}=\frac{\sqrt{1-e^{2}}}{1+e \cos (f)} \dot{f}-\frac{\sin (E)}{1-e^{2}} \dot{e}=\frac{1-e \cos (E)}{\sqrt{1-e^{2}}} \dot{f}-\frac{\sin (E)}{1-e^{2}} \dot{e} .
$$

We know that

$$
f=\theta-\omega \text {. }
$$

The true anomaly of the ascending node is $-\omega$. Let $E_{\omega}$ be the eccentric anomaly of the ascending node. Its derivative is

$$
\dot{E}_{\omega}=\frac{1-e \cos \left(E_{\omega}\right)}{\sqrt{1-e^{2}}}(-\dot{\omega})-\frac{\sin \left(E_{\omega}\right)}{1-e^{2}} \dot{e} .
$$

Let $M_{\theta}$ be the mean anomaly measured from the line of ascending node. By using Kepler's equation, it is easy to see that

$$
M_{\theta}=E-e \sin (E)-\left(E_{\omega}-e \sin \left(E_{\omega}\right)\right) .
$$

Thus, the derivative is

$$
\begin{aligned}
\dot{M}_{\theta}= & (1-e \cos (E)) \dot{E}-\left(1-e \cos \left(E_{\omega}\right)\right) \dot{E}_{\omega}-\dot{e}\left(\sin (E)-\sin \left(E_{\omega}\right)\right) \\
= & \frac{(1-e \cos (E))^{2}}{\sqrt{1-e^{2}}} \dot{\theta}-\dot{e}\left(\sin (E)-\sin \left(E_{\omega}\right)\right) \\
& -\dot{e}\left(\frac{(1-e \cos (E)) \sin (E)}{1-e^{2}}-\frac{\left(1-e \cos \left(E_{\omega}\right)\right) \sin \left(E_{\omega}\right)}{1-e^{2}}\right) \\
& -\frac{(1-e \cos (E))^{2}-\left(1-e \cos \left(E_{\omega}\right)\right)^{2}}{\sqrt{1-e^{2}}} \dot{\omega} .
\end{aligned}
$$

We know that

$$
(1-e \cos (E))=\frac{r}{a}
$$

Therefore, 


$$
\frac{(1-e \cos (E))^{2}-\left(1-e \cos \left(E_{\omega}\right)\right)^{2}}{\sqrt{1-e^{2}}}=\frac{r^{2}-r_{\omega}^{2}}{a^{2} \sqrt{1-e^{2}}} .
$$

Because of Equation (136),

$$
\begin{aligned}
\frac{r^{2}-r_{\omega}^{2}}{a^{2} \sqrt{1-e^{2}}} \dot{\omega}= & -\frac{r^{2}-r_{\omega}^{2}}{\mu a^{2} e \sqrt{1-e^{2}}}\left(\left(\frac{\partial A}{\partial p}\right)^{T} \hat{A}_{\perp} \cdot u\right) \\
& -\frac{r^{2}-r_{\omega}^{2}}{a^{2} \sqrt{1-e^{2}}} \frac{\cos (I)}{h \sin (I)}\left(\frac{\partial l}{\partial p}\right)^{T} \hat{n} \cdot u .
\end{aligned}
$$

Since we already know that

$$
\begin{aligned}
& \dot{\theta}=\frac{h}{r^{2}}-\dot{\Omega} \cos (I) \\
& \dot{e}=\frac{1}{\mu}\left(\frac{\partial A}{\partial p}\right)^{T} \hat{A} \cdot u(t),
\end{aligned}
$$

substitute the derivatives in (149) from (152) and (153), to obtain

$$
\begin{aligned}
\dot{M}_{\theta}=n & -\frac{r^{2}}{a^{2} \sqrt{1-e^{2}}} \frac{\cos (I)}{h \sin (I)}\left(\frac{\partial l}{\partial p}\right)^{T} \hat{n} \cdot u \\
& -\left(\sin (E)-\sin \left(E_{\omega}\right)+\frac{r \sin (E)-r_{\omega} \sin \left(E_{\omega}\right)}{a\left(1-e^{2}\right)}\right) \frac{1}{\mu}\left(\frac{\partial A}{\partial p}\right)^{T} \hat{A} \cdot u \\
& -\frac{r^{2}-r_{\omega}^{2}}{\mu a^{2} e \sqrt{1-e^{2}}}\left(\left(\frac{\partial A}{\partial p}\right)^{T} \hat{A} \perp \cdot u\right) \\
& +\frac{r^{2}-r_{\omega}^{2}}{a^{2} \sqrt{1-e^{2}}} \frac{\cos (I)}{h \sin (I)}\left(\frac{\partial l}{\partial p}\right)^{T} \hat{n} \cdot u,
\end{aligned}
$$

which simplifies to

$$
\begin{aligned}
\dot{M}_{\theta}=n & -\frac{r_{\omega}^{2}}{a^{2} \sqrt{1-e^{2}}} \frac{\cos (I)}{h \sin (I)}\left(\frac{\partial l}{\partial p}\right)^{T} \hat{n} \cdot u \\
& -\left(\sin (E)-\sin \left(E_{\omega}\right)+\frac{r \sin (E)-r_{\omega} \sin \left(E_{\omega}\right)}{a\left(1-e^{2}\right)}\right) \frac{1}{\mu}\left(\frac{\partial A}{\partial p}\right)^{T} \hat{A} \cdot u \\
& +\frac{r^{2}-r_{\omega}^{2}}{\mu a^{2} e \sqrt{1-e^{2}}}\left(\left(\frac{\partial A}{\partial p}\right)^{T} \hat{A}_{\perp} \cdot u\right) .
\end{aligned}
$$

